\title{
The Gaia space astrometry mission \\ Status and complementarity with radio astrometry
}

\section{François Mignard*}

Laboratoire Lagrange, Université de Nice-Sophia Antipolis, CNRS, Observatoire de la Côte

d'Azur

E-mail: francois.mignardeoca.eu

The next ESA space astrometry mission Gaia is due for launch in December 2013. Gaia will survey the entire sky and repeatedly observe the brightest one billion objects, down to 20-th magnitude, during its 5-year lifetime, pinpointing positions and parallaxes to $10 \mu$ as for stars brighter than 12-th magnitude, $25 \mu$ as for at 15-th magnitude, and $300 \mu$ as at magnitude 20. About 500,000 optically bright QSOs will be also observed with comparable accuracy allowing to build an inertial frame in the optical domain. In addition to astrometry Gaia's science data comprises broad-band photometry, and low-resolution spectro-photometry. Spectroscopic data with a resolving power of 11,500 will be obtained for the brightest 150 million sources, down to 17-th magnitude. I summarise in this paper the mission motivations and the scientific expectations of the astronomical community and pay special attention to the complementarity goals between optical and radio astrometry.

11th European VLBI Network Symposium \& Users Meeting,

October 9-12, 2012

Bordeaux, France

${ }^{*}$ Speaker. 


\section{Introduction to Gaia}

The ESA space astrometry mission Gaia is due for launch in late 2013. It will survey the sky over a period of 5 years with a sensitivity limited instrument down to the 20th magnitude in the visible range. The astrometric accuracy measured by the quality of the parallaxes is expected to be of $25 \mu$ as at $15 \mathrm{mag}$. The mission is optimised to observe stellar sources to produce a stereoscopic and kinematic census of about one billion stars in our Galaxy enabling to probe the formation and evolution of the Milky Way. The expected astrometric accuracy in position, annual proper motion and parallaxes is shown in Fig. 1 as a function of the $G$ magnitude (very similar to $R$ band).

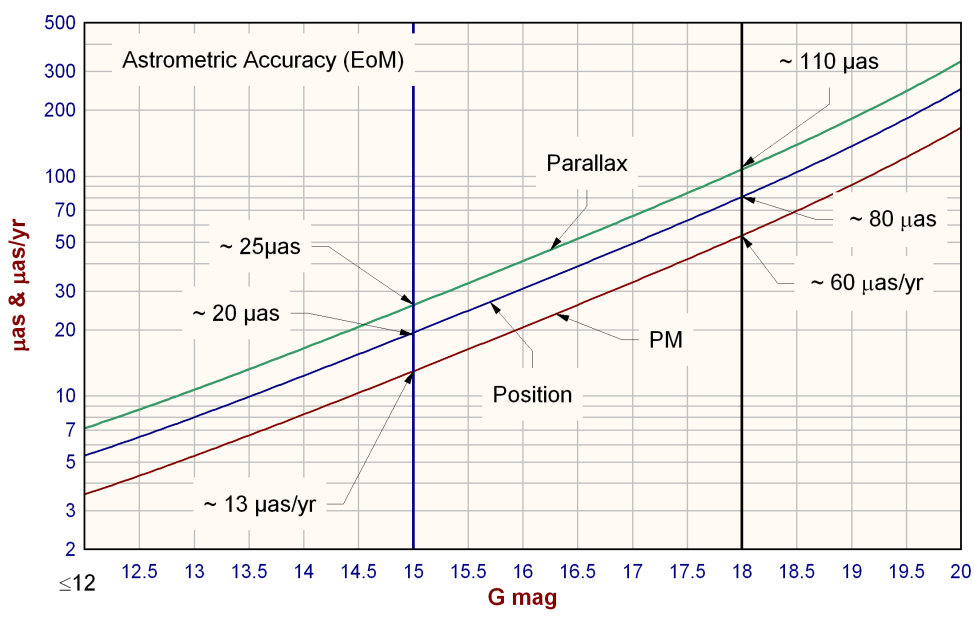

Figure 1: End-of-Mission astrometric accuracy expected with Gaia for the position, proper-motion and parallaxes, for point like sources (stars or QSOs).

The astrometric data acquisition on board of Gaia is complemented by photometric and spectroscopic information collected with two spectrophotometres and one spectrometre working synchronously with the astrometric measurements. When completed, the Gaia mission will result in an astronomical database of unprecedented breadth, accuracy and completeness, becoming available to the scientific community around 2021. This will be a reference data base for stellar and galactic physics for decades ahead. In addition to the stellar sources, the Gaia database will also include regular observations of about 400,000 moving objects from the Solar system (main belt asteroids, NEOs (Near Earth Objects), faint satellites of giant planets) and almost as many distant quasars.

Gaia will be the second space astrometry mission coming almost exactly 20 years after Hipparcos terminated its observations. It is illuminating to compare the performances between the two missions, knowing that the improvement is primarily due to the technological advances over this period. An overview of the respective performances is shown in Table 1, where one can immediately appreciate the difference in scale between the two missions. Given the lasting impact of Hipparcos in so many areas of fundamental astronomy and astrophysics [4] one can easily anticipate the broad science exploitation in galactic and stellar physics that will follow the release of the Gaia Catalogue. With a knowledge of the distance by geometric means, better than $1 \%$ for more than 10 million stars, this is the start of a new era for the calibration of the basic colour-luminosity 
Table 1: Astrometric performances of Gaia compared to Hipparcos

\begin{tabular}{lccc}
\hline & HIPPARCOS & Gaia \\
\hline Magnitude limit & 12 & $20-21$ \\
Completeness limit & $7.3-9$ & 20 & \\
Number of objects & 120000 & $35 \times 10^{6}$ & $V<15$ \\
& & $350 \times 10^{6}$ & $V<18$ \\
& & $1.3 \times 10^{9}$ & $V<20$ \\
Astrometric accuracy & 1 mas $(V<9)$ & $7 \mu$ as & $V<12$ \\
& $1-3$ mas $(V>9)$ & $25 \mu$ as & $V=15$ \\
& & $300 \mu$ as & $V=20$ \\
$\sigma_{\pi} / \pi<1 \%$ & 150 stars & $11 \times 10^{6}$ stars \\
$\sigma_{\pi} / \pi<5 \%$ & 6,200 stars & $77 \times 10^{6}$ stars \\
$\sigma_{\pi} / \pi<10 \%$ & 21,000 stars & $150 \times 10^{6}$ stars \\
Radial velocity & - & $2-10 \mathrm{~km} \mathrm{~s}{ }^{-1}$ & $V<16$ \\
Spectro-photometry & - & $\simeq 25$-colour & $V<20$ \\
Low resolution spectroscopy & - & $\mathrm{R}=11,500$ & $V<15-16$ \\
\hline
\end{tabular}

diagram and its further application in deriving distances of open or globular clusters or galaxies of the local group.

The scientific core of the Gaia mission is dedicated to stellar and galactic physics, and will bring considerable high quality data for these fields from the combination of the kinematical information allowed by astrometry and the low resolution spectroscopy more or less equivalent to photometry over the visible range with at least 15 bands. Each star will be observed about 70 times during the mission, with a slight scatter about this mean with a well defined pattern. An average sequence of observations will give two successive observations separated by just above $1.7 \mathrm{~h}$, followed by similar pairs of observations every 40 to 60 days, providing a good time sampling over the mission length of 5 years. This is required to properly sample the parallactic ellipse for disentangling the proper motion and parallax, but also for investigating more complex astrometric displacement in multiple systems or for solar system objects. A full reward of this sampling will show up also with the photometric variability analysis of at least 100 millions sources.

The sky coverage is not fully uniform, with regions of ecliptic latitudes around 45 degrees being visited more often than the ecliptic plane area. But this is an accepted compromise with limited impact on the spatial variation of the accuracy of the astrometric parameters. The sky sampling for Gaia is shown in Fig. 2 in the form of the number of passages through the fields of view for a mission of five years. The projection in galactic coordinates reveals the symmetry about the ecliptic plane (as expected with the sun being on the precession axis). The difference in sampling from place to place is significant, but not too large, since the areas with coverage much higher than average are rather narrow around ecliptic latitudes \pm 45 degrees and nearly uniform outside this zone.

Beyond the sheer measurement accuracy, the major strengths of Gaia follow from: 


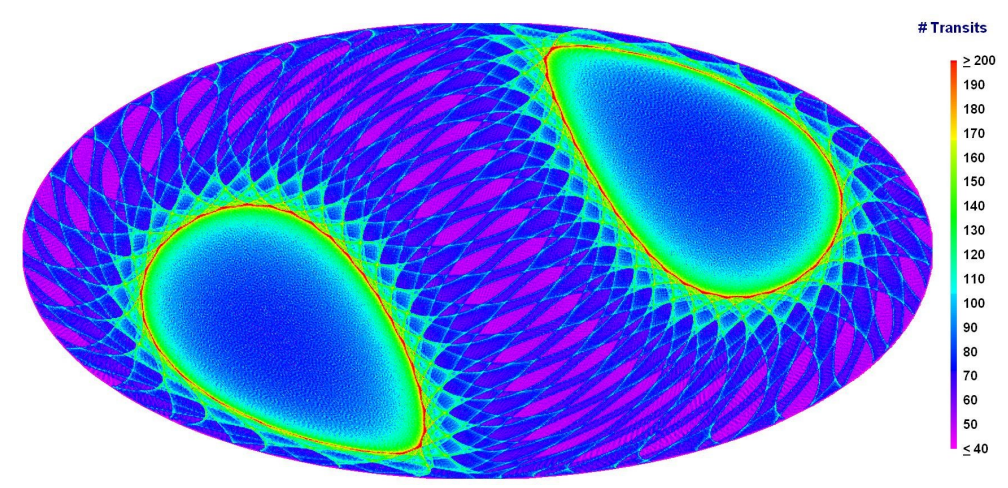

Figure 2: Average number of observations per Gaia sources as function of their position in the sky. The plot is in galactic coordinates and based on a nominal mission duration of 5 years, without dead time. The symmetry about the ecliptic plane (due to the geometry of the scanning law) is very apparent in this plot. The highest rate of observations in the two yellowish-reddish circles are centred at \pm 45 degrees of ecliptic latitude.

- its capability to perform an all-sky and sensitivity limited absolute astrometric survey

- the unique combination into a single spacecraft of the three major electronic detectors carrying out nearly contemporaneous observations in astrometry, photometry and spectroscopy,

- the huge number of objects and observations covering every kind of stars, allowing to carry out statistical analyses of deep significance.

The impact of Gaia on stellar and galactic physics can easily be surmised from the ability of space astrometry to determine accurate distances, since this is the major missing ingredient to derive accurate luminosities, ages, masses and radii. For Gaia this outstanding impact is shown in Fig. 3 giving the expected number of stars with parallax relative accuracy better than a certain limit. Compared to Hipparcos, which 20 years after the mission remains the landmark in this field, the improvement in quality and quantity is spectacular.

Almost as impressive as the astrometric accuracy at Gaia epoch is the slow aging of the Gaia catalogue as we look at its properties at times relatively far from its defining epoch. For each star, the Gaia catalogue will provide simultaneously the 5 astrometric parameters (position, proper motion components and parallax) together with their covariance matrix in equatorial coordinates. The diagonal terms will give the standard deviation at epoch, that is to say an estimate of the uncertainty. Then one can propagate this information in the past and see how good the astrometry is when positions are used, say 100 years ago. The results are shown in Fig. 4 for the sky-averaged positional accuracy between 1890-2090, which remains remarkable compared to the accuracy of the available observations done in the past. One must note the $\sim$ mas accuracy for the faint stars of the Carte du Ciel around the beginning of this program. This opens up a new window from the reprocessing of archived photographic plates or even their old measurements in case the plates are too damaged or have been lost. 


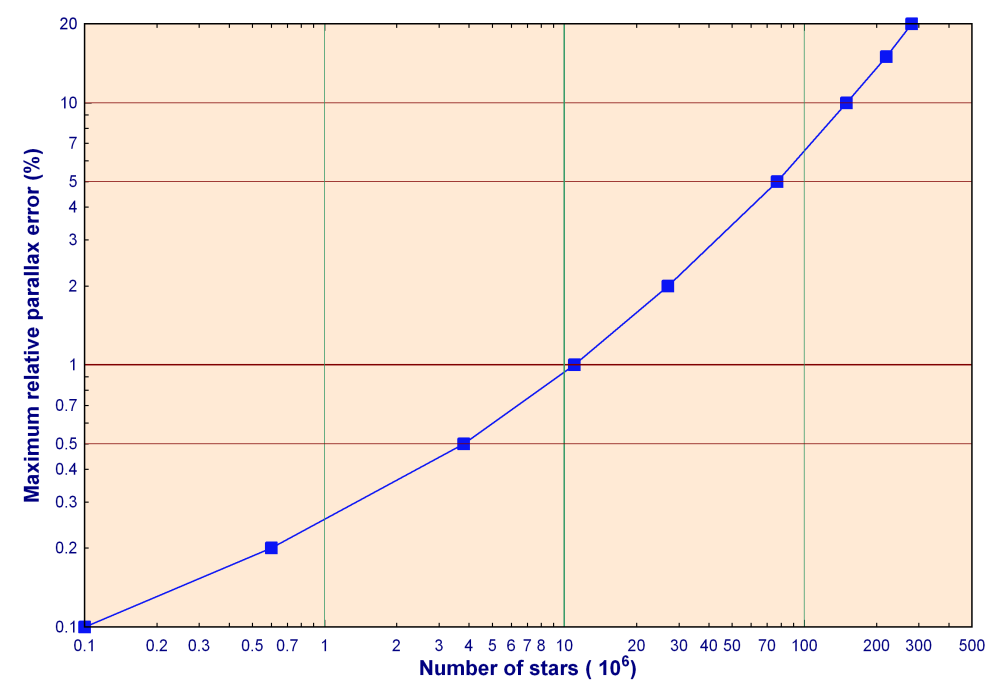

Figure 3: Relative accuracy on the parallaxes in the Gaia solution at the end of the mission. The plot gives the number of stars (abscissa) with a better parallax relative precision better than a limit given on the ordinate. For about $10^{7}$ stars the relative parallax accuracy will be better than $1 \%$. Useful to remind that Hipparcos had only 20,000 stars with $\sigma_{\pi} / \pi<10 \%$.

\section{Observations of extragalactic sources}

The sensitivity limited internal detection system will allow also to observe and determine the astrometric parameters of several 100,000s quasars, but also the fast moving objects from the solar system and other extended objects, like unresolved galaxies. These repeated observations of point-like extragalactic sources with a single instrument in space will lead to the realisation of a kinematically defined inertial frame in the optical wavelengths.

To achieve this goal one must first recognize the QSOs from the stars in an automatic and efficient way and select a clean sample of sources to serve as defining source for the frame. Then the astrometric solution for these point-like sources would not depart too much from the standard iterative process detailed in Lindegren et al. [3].

The internal photometric detection has been shown, on simulated data, very efficient to get rid of the traditional contaminants like the white dwarfs or very red stars. Final filtering with astrometry (parallaxes and proper-motions of these stars will be large and not compatible with extragalactic sources) will end up with a clean set containing virtually only point-like extragalactic sources (unresolved galaxies will fall in another box). Simultaneously photometric redshift measurements will be feasible without additional effort for most of the detected sources. Thus one may reasonably expect a census of several hundreds thousands quasars at galactic latitudes $|b|>25^{\circ}-30^{\circ}$, although these limits are not precisely known today. Closer to galactic plane, Gaia faces two difficulties: (i) the galactic extinction and reddening that will block off the light of these distant and rather faint sources, (ii) the difficulty to discriminate between the stars as their relative density to that of the quasars increases drastically at low galactic latitudes (this ratio is about 10,000 at $b=10^{\circ}$ and $G=19$ ). Multi-images formed by lensing of intervening galaxies could be detected at separa- 


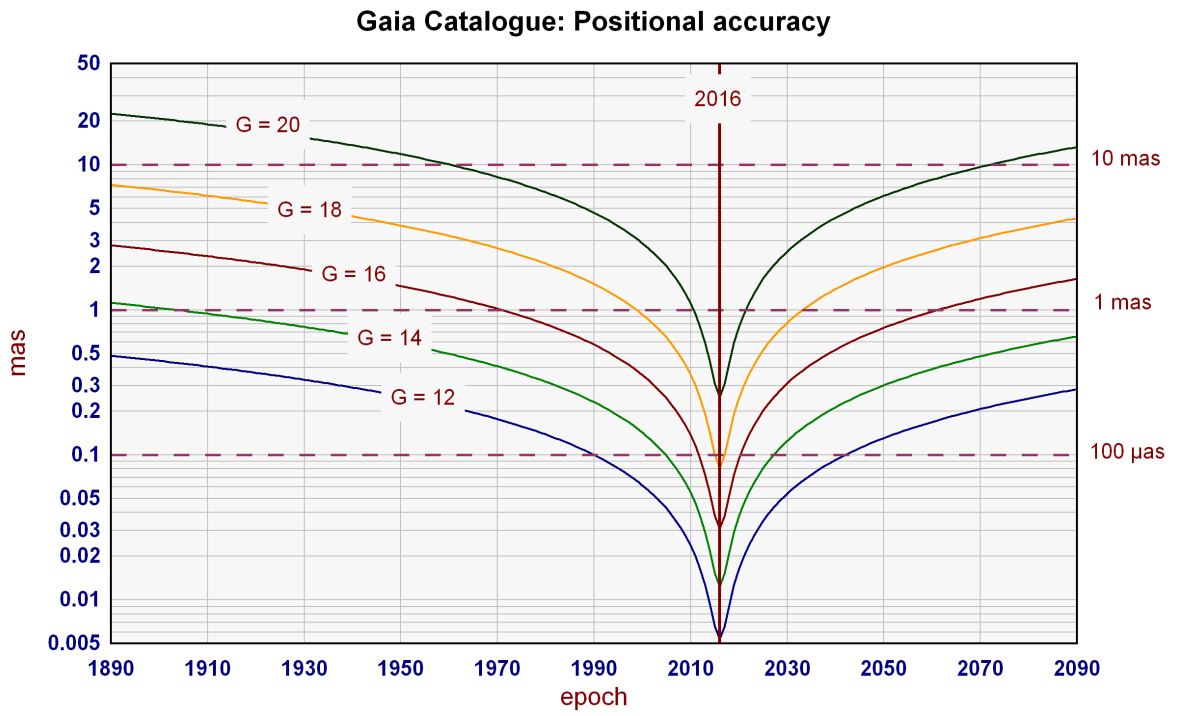

Figure 4: Evolution of the accuracy of the Gaia positional stellar catalogue with time due to the uncertainty in the annual proper motions of stars. The diagram refers only to the stars, not to the QSO-tied reference frame.

tion as small as $\sim 0.2$ arcsec, a significant improvement to the resolution of current ground based investigations with nice inferences in the distribution of distant galaxies.

From simulations based on realistic space density of optical QSOs as a function of magnitude and using the Gaia astrometric performance, Gaia should realise a quasi-inertial celestial reference frame with a residual rotation better than $0.3 \mu$ as/year. This is illustrated in Fig. 5 giving on the left scale the expected accuracy as a function of the magnitude of the faintest sources kept in the sample. The number of sources is shown on the dashed line read on the right scale. Eventually the final Gaia product directly related to the Celestial Frame will include also all the stellar sources given in the same system with accurate proper motions. The whole set will be referred to as the Gaia-CRF (Gaia Celestial Reference Frame) which will comprise:

- A set of defining sources from the clean subset of QSOs used to fix the frame spin

- A larger set of secondary QSOs not used to remove the residual rotation

- A very small set of QSOs common to Gaia and ICRF2 used to tie the orientation

- The Gaia-SRF (for Stellar Reference Frame)

a- About one billion stars with positions, proper motions and parallaxes

b- An average density of 25000 stars per square degree, highly variable with galactic latitude

The whole system will be aligned to the existing ICRF by using a small set of common sources, possibly complemented by 100-200 new sources (not in the ICRF2) being observed in the VLBI for this purpose ([1] -[2]) 


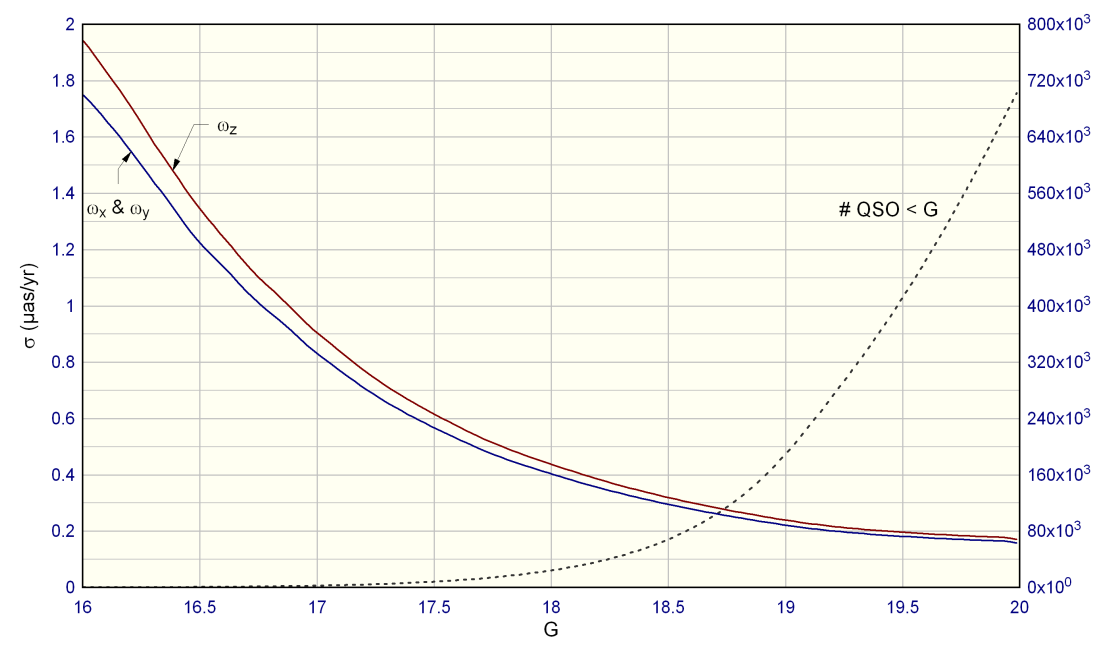

Figure 5: Inertiality of the final Gaia Celestial Reference Frame based on the QSO observations, expressed by the accuracy of the residual rotation in galactic coordinates, compared to an ideal non-rotating frame. The precision for a $G$ magnitude is computed with only sources brighter than $G$. A random instability has

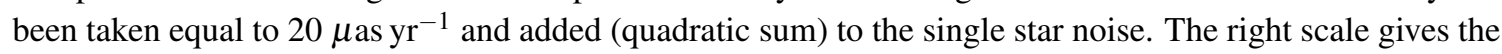
number of sources found brighter than $G$.

At the same time the Gaia initial frame derived from the fitting of the astrometric model to the observations will be tied to the non-rotating QSOs, the so-called secular drift (or galactic aberration, dipole motion, systematic glide, ...) will be computed. This will give the components of the acceleration of the solar system with respect to the frame materialised by the extragalactic sources. The main source of this effect will normally be the acceleration of the solar system towards the galactic centre nearly. The induced proper motion on QSOs will show up with a very distinctive pattern (Fig. 6) and an amplitude of about $4.5 \mu$ as/yr. With Gaia the acceleration should be determined with at least a 10-sigma accuracy.

\section{Complementarity with VLBI}

\subsection{For the Reference Frame}

As mentioned above, the astrometric solution of Gaia will use a large number of extragalactic sources to produce an inertial orientation of the reference axes in agreement with the ICRS prescriptions. This won't be a tie to the existing radio-ICRF, unlike with Hipparcos, but the production of a new realisation of the frame, without reference to a previous solution. However the orientation at the reference epoch will be adjusted to fit the existing ICRF orientation for metrological continuity. But unlike the spin, this has no deep physical significance. Therefore around Gaia mid-mission an already very accurate ICRF in optical wavelengths will be available agreeing with ICRF principles. Given its easy access through the Gaia stellar catalogue over a wide range of magnitudes, it will be used to reduce new or re-reduce old observations with the results referred to as being in the ICRF, although this won't be correct from the metrological point of view. Both radio and optical ICRF will be useful and necessary for different categories of users and a clarification should be 


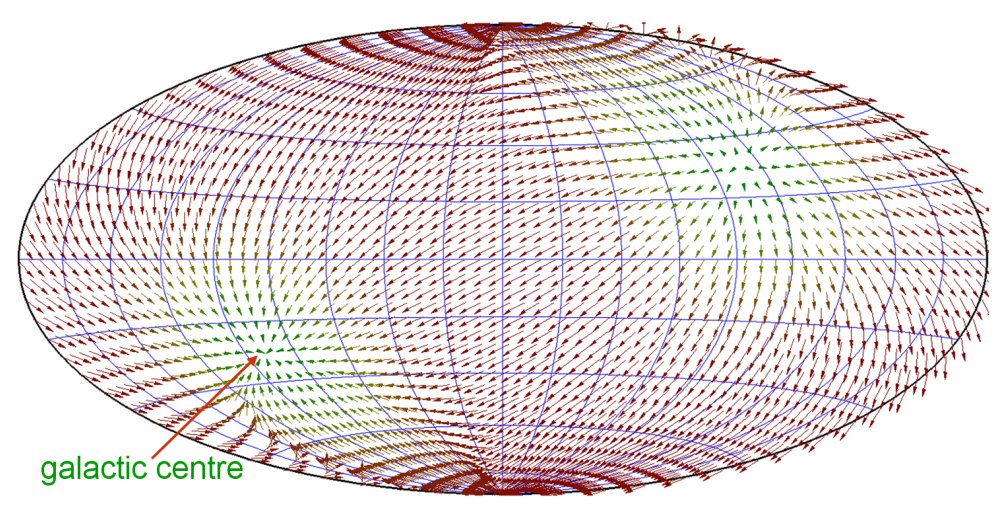

Figure 6: The so-called 'galactic aberration' or secular drift on extragalactic sources seen as a spurious proper motion with a well defined pattern with respect to the galactic center. The largest arrows 90 deg away from the galactic centre have a size of $4.5 \mu$ as/yr. Gaia will determine the three parameters of these field (dipole orientation and module) to within $0.3 \mu \mathrm{as} / \mathrm{yr}$

provided to them before the terminology turned into a mess. This will be the work of the IAU recently formed WG within the IAU division A.

\subsection{For astrometry in general}

In the following I have attempted to compare the characteristics and capabilities of both the optical and VLBI astrometry in wide or small fields. This is a first attempt and the contribution of experts in each domain would help fill this table in a more comprehensive manner. For the optical astrometry I have only considered the space astrometry which is the only solution to achieve high accuracy for the reference frame and interferometry for specific goals in very small fields, although this technique is still short from its promises in term of accuracy, with the exception of the imaging and stellar diameter measurements. Even in differential astrometry over fields larger than 10 arcsec, the instrumental calibration and the knowledge of the baseline (about $50 \mu \mathrm{m}$ required over 100$200 \mathrm{~m}$ ) limit the accuracy to about $100 \mu$ as on VLBI/PRIMA (Phase Referenced Imaging and Microarcsecond Astrometry), a factor nearly 10 from the best expectations. 


\section{Main features of optical and radio astrometry}

\section{Optical Astrometry}

- Dedicated instrumentation

- Work on survey mode over a sensitivity limited detection, leading to a very large number of sources for space astrometry.

- Global astrometry is performed by the instrument design to work in space

- For the moment only in the visible or near infra-red, but could go redder in future mission

- Accuracy in the directions between 10 to $200 \mu$ as with Gaia

- Small fields achievable primarily with interferometry (very small indeed 5 to 10 mas) but not with Gaia, but they remain limited to few bright sources in the $\mathrm{K}$ band

- No significant imaging capabilities with Gaia ( 200 mas resolution) but excellent with optical or IR interferometry.

- Extreme rigidity in the program with scanning satellites. Better with point and stare systems.

\section{VLBI}

- Multi-purpose instrumentation, implying booking for time allocation with proposals.

- Few thousands sources currently monitored for high-precision astrometry

- Earth-based instruments and global astrometric solution results from the data analysis

- By construction the instrumentation works on radio-loud sources

- Very accurate directions better than $100 \mu$ as with few $1000 \mathrm{~s} \mathrm{~km}$ baselines

- Works always in relatively small fields and connects them with a global solution

- Particularly well suited for high resolution mapping of extended sources wit sub-mas resolution

- Program flexibility to select targets and acquisition time 


\section{Conclusion}

By the end of 2013 we will know with near perfect certainty whether Gaia will be in situation to meet the great expectations of the astronomical community. The commissioning should approach its completion, just preceding the start of the routine science operation in the early 2014. The nominal mission will last for 5 years (plus a possible extension of $1 \mathrm{yr}$ ) and an additional 2 years must be allowed to finish the data processing and make the full Gaia product accessible to the astronomers. However ESA and the Gaia scientists have agreed on several intermediate deliveries compatible with the tight schedule of the data processing. From this plan one should have less than 2 years after launch a first full-sky position catalogue with unseen accuracy so far. Within this same timescale the proper motions of the Hipparcos stars will be revisited and should yield accuracies better than $50 \mu$ as/yr. Six months later the first complete astrometric catalogue (5 parameters, single stars) should be available together with the first realisation of the QSOs-based reference frame in the visible. Although this won't be yet the final accuracy, this would already be extremely valuable.

\section{References}

[1] Bourda, G., Charlot, P. et al., 2010, VLBI observations of optically-bright extragalactic radio sources for the alignment of the radio frame with the future Gaia frame I, Astrom. Astrophys. vol. 520.

[2] Bourda, G., Collioud, A. et al., 2011, VLBI observations of optically-bright extragalactic radio sources for the alignment of the radio frame with the future Gaia frame II, Astrom. Astrophys. vol. 526.

[3] Lindegren, L., Lammers, U. et al., 2012, The astrometric core solution for the Gaia mission. Overview of models, algorithms, and software implementation, Astrom. Astrophys. vol. 538.

[4] Perryman, M., 2009, Astronomical Applications of Astrometry, Cambridge Univ. Press. 